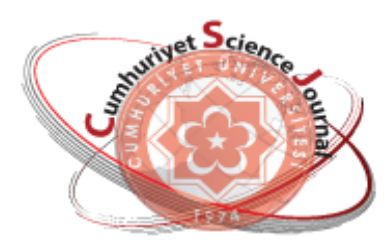

e-ISSN: 2587-246X

ISSN: 2587-2680

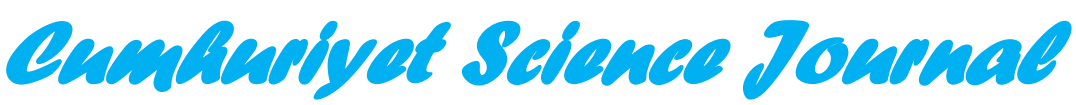

esj

Cumhuriyet Sci. J., Vol.38-4 (2017) 626-638

\title{
Some New Time Scales Weighted Ostrowski-Grüss Type Inequalities
}

\author{
Adnan TUNA
}

Nigde Omer Halisdemir University, Art and Science Faculty, Mathematics Department, 51240, Niğde / TURKEY

\begin{abstract}
In this paper, we obtain some new weighted Ostrowski-Grüss type inequalities on time scales. We also give some other interesting inequalities as special cases.

2010 Mathematics Subject Classifications : 26D15; 26E70.

Keywords: Montgomery Identitiy, Ostrowski-Grüss Type Inequalities, Time Scales.
\end{abstract}

\section{Zaman Skalasında Bazı Yeni Ağırlıklı Ostrowski-Grüss Tipi Eşitsizlikler}

Özet: Bu makalede, zaman skalasında bazı yeni ağırlıklı Ostrowski-Grüss tipi eşitsizlik elde edilmiştir. Buna ilaveten özel durumlar olarak bazı diğer ilgili eşitsizlikler verilmiştir.

Anahtar Kelimeler: Montgomery Eşitliği, Ostrowski-Grüss Tipi Eşitsizlikler, Zaman Skalası.

\section{INTRODUCTION}

In 1938, Ostrowski [11] proved the following classical Ostrowski inequality.

Theorem 1.1 Let $f:[a, b] \rightarrow R$ be continuous on $[a, b]$ and differentiable in $(a, b)$ and its derivative $f^{\prime}:(a, b) \rightarrow R$ is bounded in $(a, b)$. Then for any $x \in[a, b]$, we have

$$
\left|f(x)-\frac{1}{b-a} \int_{a}^{b} f(t) d t\right| \leq\left(\frac{1}{4}+\frac{\left(x-\frac{a+b}{2}\right)^{2}}{(b-a)^{2}}\right)(b-a)\left\|f^{\prime}\right\|_{\infty},
$$

where $\left\|f^{\prime}\right\|_{\infty}:=\sup _{x \in(a, b)}\left|f^{\prime}(x)\right|<\infty$. This inequality is sharp in the sense that the constant $\frac{1}{4}$ cannot be replaced by a smaller one.

Using the Montgomery identity on time scales, Bohner and Matthews [4] obtained the time scales Ostrowski inequality. 
The following inequality is Ostrowski-Grüss type integral inequality given by Dragomir and Wang [5] in 1997.

Theorem 1.2 Let $f:[a, b] \rightarrow R$ be continuous on $[a, b]$ and differentiable in $(a, b)$. If $\gamma \leq f^{\prime}(x) \leq \Gamma$ for all $x \in[a, b]$ for some $\gamma, \Gamma \in R$, then, for all $x \in[a, b]$, we have

$\left|f(x)-\frac{1}{b-a} \int_{a}^{b} f(t) d t-\frac{f(b)-f(a)}{b-a}\left(x-\frac{a+b}{2}\right)\right| \leq \frac{1}{4}(b-a)(\Gamma-\gamma)$,

In order to unify continuous and discrete analysis, S. Hilger [6] introduced the time scales theory in 1988. For some Ostrowski-Grüss type inequalities on time scales, see the papers $[8,9,12,13]$ where further references are provided.

In the present paper, we give the weighted Ostrowski-Grüss type inequalities on time scales and apply our results to the continuous, discrete and quantum calculus cases.

\section{GENERAL DEFINITIONS}

We briefly introduce the time scales elements in this section and refer the reader to Hilger's Ph.D. thesis [6], the books [2,3,7], and the survey [1] for proofs and further details.

Definition 2.1 A time scale $T$ is a nonempty closed subset of $R$.

We assume that $T$ has the topology that is inherited from the standard topology on $R$. The interval $[a, b]$ in $T$ means the set $[a, b]:=\{t \in T: a \leq t \leq b\}$ for the points $a<b$ in $T$.

Definition 2.2 The forward jump operator $\sigma: T \rightarrow T$ by $\sigma(t)=\inf \{s \in T: s>t\}$, while the backward jump operator $\rho: T \rightarrow T$ is defined by $\rho(t)=\sup \{s \in T: s<t\}$.

Definition 2.3 A point $t \in T$ is called right-scattered, left-scattered, right-dense and left-dense if $\sigma(t)>t$ $, \rho(t)<t, \sigma(t)=t$ and $\rho(t)=t$, respectively. Points that are both right-dense and left-dense are called dense.

Definition 2.4 The graininess function $\mu: T \rightarrow[0, \infty)$ is defined by $\mu(t)=\sigma(t)-t$ for $t \in T$. The set $T^{k}$ is defined as follows: if $T$ has a left-scattered maximum $m$, then $T^{k}=T-\{m\}$; otherwise, $T^{k}=T$.

If $T=R$, then $\mu(t)=0$, and when $T=Z$, we have $\mu(t)=1$.

Definition 2.5 Let $f: T \rightarrow R$ and fix $t \in T^{k}$. Then we define $f^{\Delta}(t) \in R$ to be the number (provided it exists) with the property that for any given $\varepsilon>0$ there exists a neighborhood $U$ of $t$ such that

$$
\left|f(\sigma(t))-f(s)-f^{\Delta}(t)[\sigma(t)-s]\right| \leq \varepsilon|\sigma(t)-s|, \quad \forall s \in U .
$$

We call $f^{\Delta}(t)$ the delta derivative of $f(t)$ at $t$.

If $T=R$, then $f^{\Delta}(t)=\frac{d f(t)}{d t}$. In the case $T=Z, f^{\Delta}(t)=\Delta f(t)=f(t+1)-f(t)$, that is, is the usual forward difference operator. 
Theorem 2.6 Assume $f, g: T \rightarrow R$ are differentiable at $t \in T^{k}$. Then the product $f g: T \rightarrow R$ is differentiable at $t$ with

$$
(f g)^{\Delta}(t)=f^{\Delta}(t) g(t)+f(\sigma(t)) g^{\Delta}(t)
$$

Definition 2.7 The function $f: T \rightarrow R$ is said to be rd-continuous (denote $f \in C_{r d}(T, \mathrm{R})$ ) on $T$ provided it is continuous at all right-dense points $t \in T$ and its left-sided limits exist at all left-dense points $t \in T$. It follows from [2,Theorem 1.74] that every rd-continuous function has an anti-derivative.

Definition 2.8 Let $f \in C_{r d}(T, \mathrm{R})$. Then $F: T^{k} \rightarrow R$ is called a delta-antiderivative of $f$ on $T$ if it satisfies $F^{\Delta}(t)=f(t)$ for all $t \in T^{k}$. In this case we define the Cauchy integral

$$
\int_{a}^{b} f(s) \Delta s=F(b)-F(a), \quad a, b \in T
$$

Theorem 2.9 Let $f, g$ be rd-continuous, $a, b, c \in T$ and $\alpha, \beta \in R$. Then

$$
\begin{aligned}
& \text { (1) } \int_{a}^{b}[\alpha f(t)+\beta g(t)] \Delta t=\alpha \int_{a}^{b} f(t) \Delta t+\beta \int_{a}^{b} g(t) \Delta t, \\
& \text { (2) } \int_{a}^{b} f(t) \Delta t=-\int_{b}^{a} f(t) \Delta t, \\
& \text { (3) } \int_{a}^{b} f(t) \Delta t=\int_{a}^{c} f(t) \Delta t+\int_{c}^{b} f(t) \Delta t, \\
& \text { (4) } \int_{a}^{b} f(t) g^{\Delta}(t) \Delta t=(f g)(b)-(f g)(a)-\int_{a}^{b} f^{\Delta}(t) g(\sigma(t)) \Delta t,
\end{aligned}
$$

Theorem 2.10 If $f$ is $\Delta$-integrable on $[a, b]$, then so is $|f|$, and

$$
\left|\int_{a}^{b} f(t) \Delta t\right| \leq \int_{a}^{b}|f(t)| \Delta t .
$$

Definition 2.11 Let $h_{k}: T^{2} \rightarrow R, k \in N_{0}$, be defined by $h_{0}(t, s)=1$, for all $s, t \in T$ and then recursively by $h_{k+1}(t, s)=\int_{s}^{t} h_{k}(\tau, s) \Delta \tau$, for all $s, t \in T$. 


\section{MAIN RESULTS}

For the proof our main results, we will need the following lemma due to Nwaeze [10].

Lemma 3.1 (A Weighted generalized Montgomery Identity) Let $v:[a, b] \rightarrow[0, \infty$ ) be rd-continuous and positive and $w:[a, b] \rightarrow R$ be differentiable such that $w^{\Delta}(t)=v(t)$ on $[a, b]$. Suppose also that $a, b, t, x \in T, a<b, f:[a, b] \rightarrow R$ is differentiable, and that $\psi$ is a function of $[0,1]$ into $[0,1]$. Then we have

$$
\begin{aligned}
& {\left[\frac{1+\psi(1-\lambda)-\psi(\lambda)}{2} f(x)+\frac{\psi(\lambda) f(a)+(1-\psi(1-\lambda)) f(b)}{2}\right] \int_{a}^{b} v(t) \Delta t} \\
& =\int_{a}^{b} v(t) f(\sigma(t)) \Delta t+\int_{a}^{b} K(x, t) f^{\Delta}(t) \Delta t,
\end{aligned}
$$

where

$$
K(x, t)= \begin{cases}w(t)-\left(w(a)+\psi(\lambda) \frac{w(b)-w(a)}{2}\right), & t \in[a, x), \\ w(t)-\left(w(a)+(1+\psi(1-\lambda)) \frac{w(b)-w(a)}{2}\right), & t \in[x, b] .\end{cases}
$$

The following inequalities are the weighted Ostrowski-Grüss type inequalities on time scales.

Theorem 3.2 Let $v:[a, b] \rightarrow[0, \infty)$ be rd-continuous and positive and $w:[a, b] \rightarrow R$ be differentiable such that $w^{\Delta}(t)=v(t)$ on $[a, b]$. Suppose that $a, b, t, x \in T, a<b, f:[a, b] \rightarrow R$ is differentiable, and that $\psi$ is a function of $[0,1]$ into $[0,1]$. Then for all $x \in[a, b]$, we have

$$
\begin{aligned}
& \mid \frac{1}{b-a}\left[\frac{1+\psi(1-\lambda)-\psi(\lambda)}{2} f(x)+\frac{\psi(\lambda) f(a)+(1-\psi(1-\lambda)) f(b)}{2}\right]_{a}^{b} v(t) \Delta t \\
& -\frac{1}{b-a} \int_{a}^{b} v(t) f(\sigma(t)) \Delta t-\frac{f(b)-f(a)}{(b-a)^{2}} \int_{a}^{b} K(x, t) \Delta t \mid \\
& \leq\left[\frac{1}{b-a} \int_{a}^{b} K^{2}(x, t) \Delta t-\left(\frac{1}{b-a} \int_{a}^{b} K(x, t) \Delta t\right)^{2}\right]^{\frac{1}{2}}\left[\frac{1}{b-a} \int_{a}^{b}\left(f^{\Delta}(t)\right)^{2} \Delta t-\left(\frac{f(b)-f(a)}{b-a}\right)^{2}\right]^{\frac{1}{2}},
\end{aligned}
$$

where 


$$
K(x, t)= \begin{cases}w(t)-\left(w(a)+\psi(\lambda) \frac{w(b)-w(a)}{2}\right), & t \in[a, x), \\ w(t)-\left(w(a)+(1+\psi(1-\lambda)) \frac{w(b)-w(a)}{2}\right), & t \in[x, b] .\end{cases}
$$

Proof. We have

$$
\begin{aligned}
& \frac{1}{b-a} \int_{a}^{b} K(x, t) f^{\Delta}(t) \Delta t-\left(\frac{1}{b-a} \int_{a}^{b} K(x, t) \Delta t\right)\left(\frac{1}{b-a} \int_{a}^{b} f^{\Delta}(t) \Delta t\right) \\
& =\frac{1}{2(b-a)^{2}} \int_{a}^{b} \int_{a}^{b}(K(x, t)-K(x, \mathrm{~s}))\left(f^{\Delta}(t)-f^{\Delta}(s)\right) \Delta t \Delta s,
\end{aligned}
$$

From the hypothesis of Lemma 3.1, we have (see also [10])

$$
\begin{aligned}
& \int_{a}^{b} K(x, t) f^{\Delta}(t) \Delta t=\left[\frac{1+\psi(1-\lambda)-\psi(\lambda)}{2} f(x)+\frac{\psi(\lambda) f(a)+(1-\psi(1-\lambda)) f(b)}{2}\right] \int_{a}^{b} v(t) \Delta t \\
& -\int_{a}^{b} v(t) f(\sigma(t)) \Delta t,
\end{aligned}
$$

and

$$
\frac{1}{b-a} \int_{a}^{b} f^{\Delta}(t) \Delta t=\frac{f(b)-f(a)}{b-a}
$$

Using the Cauchy-Schwartz inequality, we have

$$
\begin{aligned}
& \left|\frac{1}{2(b-a)^{2}} \int_{a}^{b} \int_{a}^{b}(K(x, t)-K(x, \mathrm{~s}))\left(f^{\Delta}(t)-f^{\Delta}(s)\right) \Delta t \Delta s\right| \\
& \leq\left(\frac{1}{2(b-a)^{2}} \int_{a}^{b} \int_{a}^{b}(K(x, t)-K(x, \mathrm{~s}))^{2} \Delta t \Delta s\right)^{\frac{1}{2}}\left(\frac{1}{2(b-a)^{2}} \int_{a}^{b} \int_{a}^{b}\left(f^{\Delta}(t)-f^{\Delta}(s)\right)^{2} \Delta t \Delta s\right)^{\frac{1}{2}},
\end{aligned}
$$

However

$$
\frac{1}{2(b-a)^{2}} \int_{a}^{b} \int_{a}^{b}(K(x, t)-K(x, \mathrm{~s}))^{2} \Delta t \Delta s=\frac{1}{b-a} \int_{a}^{b} K^{2}(x, t) \Delta t-\left(\frac{1}{b-a} \int_{a}^{b} K(x, t) \Delta t\right)^{2},
$$

and

$$
\frac{1}{2(b-a)^{2}} \int_{a}^{b} \int_{a}^{b}\left(f^{\Delta}(t)-f^{\Delta}(s)\right)^{2} \Delta t \Delta s=\frac{1}{b-a} \int_{a}^{b}\left(f^{\Delta}(t)\right)^{2} \Delta t-\left(\frac{1}{b-a} \int_{a}^{b} f^{\Delta}(t) \Delta t\right)^{2},
$$

Using (6)-(11), then the proof of inequality (5) is completed. 
Corollary 3.3 In case of the $T=R$ in Theorem 3.2, we have

$$
\begin{aligned}
& \mid \frac{1}{b-a}\left[\frac{1+\psi(1-\lambda)-\psi(\lambda)}{2} f(x)+\frac{\psi(\lambda) f(a)+(1-\psi(1-\lambda)) f(b)}{2}\right] \int_{a}^{b} v(t) d t \\
& -\frac{1}{b-a} \int_{a}^{b} v(t) f(t) d t-\frac{f(b)-f(a)}{(b-a)^{2}} \int_{a}^{b} K(x, t) d t \mid \\
& \leq\left[\frac{1}{b-a} \int_{a}^{b} K^{2}(x, t) d t-\left(\frac{1}{b-a} \int_{a}^{b} K(x, t) d t\right)^{2}\right]^{\frac{1}{2}}\left[\frac{1}{b-a} \int_{a}^{b}\left(f^{\prime}(t)\right)^{2} d t-\left(\frac{f(b)-f(a)}{b-a}\right)^{2}\right]^{\frac{1}{2}},
\end{aligned}
$$

where $w^{\prime}(t)=v(t)$ on $[a, b]$ and

$$
K(x, t)= \begin{cases}w(t)-\left(w(a)+\psi(\lambda) \frac{w(b)-w(a)}{2}\right), & t \in[a, x), \\ w(t)-\left(w(a)+(1+\psi(1-\lambda)) \frac{w(b)-w(a)}{2}\right), & t \in[x, b] .\end{cases}
$$

Corollary 3.4 In case of the $T=Z$ in Theorem 3.2, we have

$$
\begin{aligned}
& \mid \frac{1}{b-a}\left[\frac{1+\psi(1-\lambda)-\psi(\lambda)}{2} f(x)+\frac{\psi(\lambda) f(a)+(1-\psi(1-\lambda)) f(b)}{2}\right] \sum_{t=a}^{b-1} v(t) \\
& -\frac{1}{b-a} \sum_{t=a}^{b-1} v(t) f(t+1)-\frac{f(b)-f(a)}{(b-a)^{2}} \sum_{t=a}^{b-1} K(x, t) \mid \\
& \leq\left[\frac{1}{b-a} \sum_{t=a}^{b-1} K^{2}(x, t)-\left(\frac{1}{b-a} \sum_{t=a}^{b-1} K(x, t)\right)^{2}\right]^{\frac{1}{2}}\left[\frac{1}{b-a} \sum_{t=a}^{b-1}(\Delta f(t))^{2}-\left(\frac{f(b)-f(a)}{b-a}\right)^{2}\right]^{\frac{1}{2}},
\end{aligned}
$$

where $v(t)=w(t+1)-w(t)$ on $[a, b-1]$ and

$$
K(x, t)= \begin{cases}w(t)-\left(w(a)+\psi(\lambda) \frac{w(b)-w(a)}{2}\right), & t \in[a, x-1), \\ w(t)-\left(w(a)+(1+\psi(1-\lambda)) \frac{w(b)-w(a)}{2}\right), & t \in[x, b-1] .\end{cases}
$$

Corollary 3.5 Let $T=q^{N_{0}}$, with $q>1, a=q^{m}$ and $b=q^{n}$ with $m<n$ in Theorem 3.2. Then we have

$$
\mid \frac{1}{q^{n}-q^{m}}\left[\frac{1+\psi(1-\lambda)-\psi(\lambda)}{2} f(x)+\frac{\psi(\lambda) f\left(q^{m}\right)+(1-\psi(1-\lambda)) f\left(q^{n}\right)}{2}\right] \int_{q^{m}}^{q^{n}} v(t) d_{q} t
$$




$$
\begin{aligned}
& -\frac{1}{q^{n}-q^{m}} \int_{q^{m}}^{q^{n}} v(t) f(q t) d_{q} t-\frac{f\left(q^{n}\right)-f\left(q^{m}\right)}{\left(q^{n}-q^{m}\right)^{2}} \sum_{k=m}^{n-1} K\left(x, q^{k}\right) \mid \\
& \leq\left[\frac{1}{q^{n}-q^{m}} \sum_{k=m}^{n-1} K^{2}\left(x, q^{k}\right)-\left(\frac{1}{q^{n}-q^{m}} \sum_{k=m}^{n-1} K\left(x, q^{k}\right)\right)^{2}\right]^{\frac{1}{2}} \\
& \times\left[\frac{1}{q^{n}-q^{m}} \int_{q^{m}}^{q^{n}}\left(f^{\Delta}(t)\right)^{2} d_{q} t-\left(\frac{f\left(q^{n}\right)-f\left(q^{m}\right)}{q^{n}-q^{m}}\right)^{2}\right]^{\frac{1}{2}},
\end{aligned}
$$

where $v(t)=\frac{w(q t)-w(t)}{(q-1) t}$ on $\left[q^{m}, q^{n}\right]$ and

$$
K(x, t)= \begin{cases}w\left(q^{k}\right)-\left(w\left(q^{m}\right)+\psi(\lambda) \frac{w\left(q^{n}\right)-w\left(q^{m}\right)}{2}\right), & q^{k} \in\left[q^{m}, x\right), \\ w\left(q^{k}\right)-\left(w\left(q^{m}\right)+(1+\psi(1-\lambda)) \frac{w\left(q^{n}\right)-w\left(q^{m}\right)}{2}\right), & q^{k} \in\left[x, q^{n}\right] .\end{cases}
$$

Corollary 3.6 If we take $w(t)=t$ in the Theorem 3.2, then we get

$$
\begin{aligned}
& \mid \frac{1+\psi(1-\lambda)-\psi(\lambda)}{2} f(x)+\frac{\psi(\lambda) f(a)+(1-\psi(1-\lambda)) f(b)}{2} \\
& -\frac{1}{b-a} \int_{a}^{b} f(\sigma(t)) \Delta t-\frac{f(b)-f(a)}{(b-a)^{2}}\left[-h_{2}\left(a, a+\psi(\lambda) \frac{b-a}{2}\right)+h_{2}\left(x, a+\psi(\lambda) \frac{b-a}{2}\right)\right. \\
& \left.-h_{2}\left(x, a+(1+\psi(1-\lambda)) \frac{b-a}{2}\right)+h_{2}\left(b, a+(1+\psi(1-\lambda)) \frac{b-a}{2}\right)\right] \mid \\
& \leq\left[\frac{1}{b-a}\left(\int_{a}^{x}\left[t-\left(a+\psi(\lambda) \frac{b-a}{2}\right)\right]^{2} \Delta t+\int_{x}^{b}\left[t-\left(a+(1+\psi(1-\lambda)) \frac{b-a}{2}\right)\right]^{2} \Delta t\right)\right. \\
& -\frac{1}{(b-a)^{2}}\left(-h_{2}\left(a, a+\psi(\lambda) \frac{b-a}{2}\right)+h_{2}\left(x, a+\psi(\lambda) \frac{b-a}{2}\right)\right. \\
& \left.\left.-h_{2}\left(x, a+(1+\psi(1-\lambda)) \frac{b-a}{2}\right)+h_{2}\left(b, a+(1+\psi(1-\lambda)) \frac{b-a}{2}\right)\right)^{2}\right]^{\frac{1}{2}}
\end{aligned}
$$


$\times\left[\frac{1}{b-a} \int_{a}^{b}\left(f^{\Delta}(t)\right)^{2} \Delta t-\left(\frac{f(b)-f(a)}{b-a}\right)^{2}\right]^{\frac{1}{2}}$,

for all $\lambda \in[0,1]$ such that $a+\psi(\lambda) \frac{b-a}{2}$ and $a+(1+\psi(1-\lambda)) \frac{b-a}{2}$ are in $T$,

$x \in\left[a+\psi(\lambda) \frac{b-a}{2}, a+(1+\psi(1-\lambda)) \frac{b-a}{2}\right] \cap T$.

Corollary 3.7 If we consider $\psi(\lambda)=\lambda$ in the Corollary 3.6, then we have

$$
\begin{aligned}
& \mid(1-\lambda) f(x)+\lambda \frac{f(a)+f(b)}{2}-\frac{1}{b-a} \int_{a}^{b} f(\sigma(t)) \Delta t \\
& -\frac{f(b)-f(a)}{(b-a)^{2}}\left[-h_{2}\left(a, a+\lambda \frac{b-a}{2}\right)+h_{2}\left(x, a+\lambda \frac{b-a}{2}\right)\right. \\
& \left.-h_{2}\left(x, b-\lambda \frac{b-a}{2}\right)+h_{2}\left(b, b-\lambda \frac{b-a}{2}\right)\right] \mid \\
& \leq\left[\frac{1}{b-a}\left(\int_{a}^{x}\left[t-\left(a+\lambda \frac{b-a}{2}\right)\right]^{2} \Delta t+\int_{x}^{b}\left[t-\left(b-\lambda \frac{b-a}{2}\right)\right]^{2} \Delta t\right)\right. \\
& -\frac{1}{(b-a)^{2}}\left(-h_{2}\left(a, a+\lambda \frac{b-a}{2}\right)+h_{2}\left(x, a+\lambda \frac{b-a}{2}\right)\right. \\
& \left.\left.-h_{2}\left(x, b-\lambda \frac{b-a}{2}\right)+h_{2}\left(b, b-\lambda \frac{b-a}{2}\right)\right)^{2}\right]^{\frac{1}{2}}\left[\frac{1}{b-a} \int_{a}^{b}\left(f^{\Delta}(t)\right)^{2} \Delta t-\left(\frac{f(b)-f(a)}{b-a}\right)^{2}\right]^{\frac{1}{2}},
\end{aligned}
$$

for all $\lambda \in[0,1]$ such that $a+\lambda \frac{b-a}{2}$ and $b-\lambda \frac{b-a}{2}$ are in $T, x \in\left[a+\lambda \frac{b-a}{2}, b-\lambda \frac{b-a}{2}\right] \cap T$.

Proposition 3.8 In case of the $\lambda=0$ in the Corollary 3.7, we obtain

$$
\begin{aligned}
& \left|f(x)-\frac{1}{b-a} \int_{a}^{b} f(\sigma(t)) \Delta t-\frac{f(b)-f(a)}{(b-a)^{2}}\left[h_{2}(x, a)-h_{2}(x, b)\right]\right| \\
& \leq\left[\frac{1}{b-a}\left(\int_{a}^{x}(t-a)^{2} \Delta t+\int_{x}^{b}(b-t)^{2} \Delta t\right)-\frac{1}{(b-a)^{2}}\left(h_{2}(x, a)-h_{2}(x, b)\right)^{2}\right]^{\frac{1}{2}} \\
& \times\left[\frac{1}{b-a} \int_{a}^{b}\left(f^{\Delta}(t)\right)^{2} \Delta t-\left(\frac{f(b)-f(a)}{b-a}\right)^{2}\right]^{\frac{1}{2}} .
\end{aligned}
$$


Proposition 3.9 In case of the $\lambda=\frac{1}{2}$ in the Corollary 3.7, we get

$$
\begin{aligned}
& \mid \frac{1}{2} f(x)+\frac{f(a)+f(b)}{4}-\frac{1}{b-a} \int_{a}^{b} f(\sigma(t)) \Delta t \\
& -\frac{f(b)-f(a)}{(b-a)^{2}}\left[-h_{2}\left(a, \frac{3 a+b}{4}\right)+h_{2}\left(x, \frac{3 a+b}{4}\right)-h_{2}\left(x, \frac{a+3 b}{4}\right)+h_{2}\left(b, \frac{a+3 b}{4}\right)\right] \mid \\
& \leq\left[\frac{1}{b-a}\left(\int_{a}^{x}\left(t-\frac{3 a+b}{4}\right)^{2} \Delta t+\int_{x}^{b}\left(t-\frac{a+3 b}{4}\right)^{2} \Delta t\right)\right. \\
& \left.-\frac{1}{(b-a)^{2}}\left(-h_{2}\left(a, \frac{3 a+b}{4}\right)+h_{2}\left(x, \frac{3 a+b}{4}\right)-h_{2}\left(x, \frac{a+3 b}{4}\right)+h_{2}\left(b, \frac{a+3 b}{4}\right)\right)^{2}\right]^{\frac{1}{2}} \\
& \times\left[\frac{1}{b-a} \int_{a}^{b}\left(f^{\Delta}(t)\right)^{2} \Delta t-\left(\frac{f(b)-f(a)}{b-a}\right)^{2}\right]^{\frac{1}{2}},
\end{aligned}
$$

for all $\lambda \in[0,1]$ such that $\frac{3 a+b}{4}$ and $\frac{a+3 b}{4}$ are in $T, x \in\left[\frac{3 a+b}{4}, \frac{a+3 b}{4}\right] \cap T$.

Theorem 3.10 Let $v:[a, b] \rightarrow[0, \infty)$ be rd-continuous and positive and $w:[a, b] \rightarrow R$ be differentiable such that $w^{\Delta}(t)=v(t)$ on $[a, b]$. Suppose that $a, b, t, x \in T, a<b, f:[a, b] \rightarrow R$ is differentiable function such that there exist constant $\gamma, \Gamma \in R$, with $\gamma \leq f^{\Delta}(x) \leq \Gamma, x \in[a, b]$ and that $\psi$ is a function of $[0,1]$ into $[0,1]$. Then for all $x \in[a, b]$, we have

$$
\begin{gathered}
\mid\left[\frac{1+\psi(1-\lambda)-\psi(\lambda)}{2} f(x)+\frac{\psi(\lambda) f(a)+(1-\psi(1-\lambda)) f(b)}{2}\right] \int_{a}^{b} v(t) \Delta t \\
\quad-\int_{a}^{b} v(t) f(\sigma(t)) \Delta t-\frac{\gamma+\Gamma}{2} \int_{a}^{b} K(x, t) \Delta t\left|\leq \frac{\Gamma-\gamma}{2} \int_{a}^{b}\right| K(x, t) \mid \Delta t,
\end{gathered}
$$

where

$$
K(x, t)= \begin{cases}w(t)-\left(w(a)+\psi(\lambda) \frac{w(b)-w(a)}{2}\right), & t \in[a, x), \\ w(t)-\left(w(a)+(1+\psi(1-\lambda)) \frac{w(b)-w(a)}{2}\right), & t \in[x, b] .\end{cases}
$$

Proof. From Lemma 3.1, we have 


$$
\begin{aligned}
& \int_{a}^{b} K(x, t) f^{\Delta}(t) \Delta t=\left[\frac{1+\psi(1-\lambda)-\psi(\lambda)}{2} f(x)+\frac{\psi(\lambda) f(a)+(1-\psi(1-\lambda)) f(b)}{2}\right]_{a}^{b} v(t) \Delta t \\
& -\int_{a}^{b} v(t) f(\sigma(t)) \Delta t,
\end{aligned}
$$

Let $C=\frac{\gamma+\Gamma}{2}$. Using (20), we get

$$
\begin{aligned}
& \int_{a}^{b} K(x, t)\left(f^{\Delta}(t)-C\right) \Delta t=\left[\frac{1+\psi(1-\lambda)-\psi(\lambda)}{2} f(x)+\frac{\psi(\lambda) f(a)+(1-\psi(1-\lambda)) f(b)}{2}\right]_{a}^{b} v(t) \Delta t \\
& -\int_{a}^{b} v(t) f(\sigma(t)) \Delta t-\frac{\gamma+\Gamma}{2} \int_{a}^{b} K(x, t) \Delta t .
\end{aligned}
$$

Taking absolute value, we get

$$
\left|\int_{a}^{b} K(x, t)\left(f^{\Delta}(t)-C\right) \Delta t\right| \leq \frac{\Gamma-\gamma}{2} \int_{a}^{b}|K(x, t)| \Delta t,
$$

From (20)-(22), we get the desired result.

Corollary 3.11 In case of the $T=R$ in Theorem 3.10, we have

$$
\begin{aligned}
& \mid\left[\frac{1+\psi(1-\lambda)-\psi(\lambda)}{2} f(x)+\frac{\psi(\lambda) f(a)+(1-\psi(1-\lambda)) f(b)}{2}\right] \int_{a}^{b} v(t) d t \\
& -\int_{a}^{b} v(t) f(t) d t-\frac{\gamma+\Gamma}{2} \int_{a}^{b} K(x, t) d t\left|\leq \frac{\Gamma-\gamma}{2} \int_{a}^{b}\right| K(x, t) \mid d t,
\end{aligned}
$$

where $w^{\prime}(t)=v(t)$ on $[a, b]$ and

$$
K(x, t)= \begin{cases}w(t)-\left(w(a)+\psi(\lambda) \frac{w(b)-w(a)}{2}\right), & t \in[a, x), \\ w(t)-\left(w(a)+(1+\psi(1-\lambda)) \frac{w(b)-w(a)}{2}\right), & t \in[x, b] .\end{cases}
$$

Corollary 3.12 In case of the $T=Z$ in Theorem 3.10, we have

$$
\begin{aligned}
& \mid\left[\frac{1+\psi(1-\lambda)-\psi(\lambda)}{2} f(x)+\frac{\psi(\lambda) f(a)+(1-\psi(1-\lambda)) f(b)}{2}\right] \sum_{t=a}^{b-1} v(t) \\
& -\sum_{t=a}^{b-1} v(t) f(t+1)-\frac{\gamma+\Gamma}{2} \sum_{t=a}^{b-1} K(x, t)\left|\leq \frac{\Gamma-\gamma}{2} \sum_{t=a}^{b-1}\right| K(x, t) \mid
\end{aligned}
$$

where $v(t)=w(t+1)-w(t)$ on $[a, b-1]$ and 


$$
K(x, t)= \begin{cases}w(t)-\left(w(a)+\psi(\lambda) \frac{w(b)-w(a)}{2}\right), & t \in[a, x-1), \\ w(t)-\left(w(a)+(1+\psi(1-\lambda)) \frac{w(b)-w(a)}{2}\right), & t \in[x, b-1] .\end{cases}
$$

Corollary 3.13 Let $T=q^{N_{0}}$, with $q>1, a=q^{m}$ and $b=q^{n}$ with $m<n$ in Theorem 3.10. Then we have

$$
\begin{aligned}
& \mid\left[\frac{1+\psi(1-\lambda)-\psi(\lambda)}{2} f(x)+\frac{\psi(\lambda) f\left(q^{m}\right)+(1-\psi(1-\lambda)) f\left(q^{n}\right)}{2}\right] \int_{q^{m}}^{q^{n}} v(t) d_{q} t \\
& -\int_{q^{m}}^{q^{n}} v(t) f(q t) d_{q} t-\frac{\gamma+\Gamma}{2} \sum_{k=m}^{n-1} K\left(x, q^{k}\right)\left|\leq \frac{\Gamma-\gamma}{2} \sum_{k=m}^{n-1}\right| K\left(x, q^{k}\right),
\end{aligned}
$$

where $v(t)=\frac{w(q t)-w(t)}{(q-1) t}$ on $\left[q^{m}, q^{n}\right]$ and

$$
K(x, t)= \begin{cases}w\left(q^{k}\right)-\left(w\left(q^{m}\right)+\psi(\lambda) \frac{w\left(q^{n}\right)-w\left(q^{m}\right)}{2}\right), & q^{k} \in\left[q^{m}, x\right), \\ w\left(q^{k}\right)-\left(w\left(q^{m}\right)+(1+\psi(1-\lambda)) \frac{w\left(q^{n}\right)-w\left(q^{m}\right)}{2}\right), & q^{k} \in\left[x, q^{n}\right] .\end{cases}
$$

Corollary 3.14 If we take $w(t)=t$ in the Theorem 3.10, then we obtain

$$
\begin{aligned}
& \mid \frac{1+\psi(1-\lambda)-\psi(\lambda)}{2} f(x)+\frac{\psi(\lambda) f(a)+(1-\psi(1-\lambda)) f(b)}{2} \\
& -\frac{1}{b-a} \int_{a}^{b} f(\sigma(t)) \Delta t-\frac{\gamma+\Gamma}{2(b-a)}\left[-h_{2}\left(a, a+\psi(\lambda) \frac{b-a}{2}\right)+h_{2}\left(x, a+\psi(\lambda) \frac{b-a}{2}\right)\right. \\
& \left.-h_{2}\left(x, a+(1+\psi(1-\lambda)) \frac{b-a}{2}\right)+h_{2}\left(b, a+(1+\psi(1-\lambda)) \frac{b-a}{2}\right)\right] \mid \\
& \leq \frac{\Gamma-\gamma}{2(b-a)}\left[h_{2}\left(a, a+\psi(\lambda) \frac{b-a}{2}\right)+h_{2}\left(x, a+\psi(\lambda) \frac{b-a}{2}\right)\right. \\
& +h_{2}\left(x, a+(1+\psi(1-\lambda)) \frac{b-a}{2}\right)+h_{2}\left(b, a+(1+\psi(1-\lambda)) \frac{b-a}{2}\right),
\end{aligned}
$$

for all $\lambda \in[0,1]$ such that $a+\psi(\lambda) \frac{b-a}{2}$ and $a+(1+\psi(1-\lambda)) \frac{b-a}{2}$ are in $T$, $x \in\left[a+\psi(\lambda) \frac{b-a}{2}, a+(1+\psi(1-\lambda)) \frac{b-a}{2}\right] \cap T$. 
Corollary 3.15 If we take $\psi(\lambda)=\lambda$ in the Corollary 3.14, then we get

$$
\begin{aligned}
& \mid(1-\lambda) f(x)+\lambda \frac{f(a)+f(b)}{2}-\frac{1}{b-a} \int_{a}^{b} f(\sigma(t)) \Delta t \\
& -\frac{\gamma+\Gamma}{2(b-a)}\left[-h_{2}\left(a, a+\lambda \frac{b-a}{2}\right)+h_{2}\left(x, a+\lambda \frac{b-a}{2}\right)-h_{2}\left(x, b-\lambda \frac{b-a}{2}\right)+h_{2}\left(b, b-\lambda \frac{b-a}{2}\right)\right] \mid \\
\leq & \frac{\Gamma-\gamma}{2(b-a)}\left[h_{2}\left(a, a+\lambda \frac{b-a}{2}\right)+h_{2}\left(x, a+\lambda \frac{b-a}{2}\right)+h_{2}\left(x, b-\lambda \frac{b-a}{2}\right)+h_{2}\left(b, b-\lambda \frac{b-a}{2}\right)\right],
\end{aligned}
$$

for all $\lambda \in[0,1]$ such that $a+\lambda \frac{b-a}{2}$ and $b-\lambda \frac{b-a}{2}$ are in $T, x \in\left[a+\lambda \frac{b-a}{2}, b-\lambda \frac{b-a}{2}\right] \cap T$.

Proposition 3.16 In case of the $\lambda=0$ in the Corollary 3.15, we get

$$
\left|f(x)-\frac{1}{b-a} \int_{a}^{b} f(\sigma(t)) \Delta t-\frac{\gamma+\Gamma}{2(b-a)}\left[h_{2}(x, a)-h_{2}(x, b)\right]\right| \leq \frac{\Gamma-\gamma}{2(b-a)}\left(h_{2}(x, a)+h_{2}(x, b)\right),
$$

Proposition 3.17 In case of the $\lambda=\frac{1}{2}$ in the Corollary 3.15, we obtain

$$
\begin{aligned}
& \mid \frac{1}{2} f(x)+\frac{f(a)+f(b)}{4}-\frac{1}{b-a} \int_{a}^{b} f(\sigma(t)) \Delta t \\
& -\frac{\gamma+\Gamma}{2(b-a)}\left[-h_{2}\left(a, \frac{3 a+b}{4}\right)+h_{2}\left(x, \frac{3 a+b}{4}\right)-h_{2}\left(x, \frac{a+3 b}{4}\right)+h_{2}\left(b, \frac{a+3 b}{4}\right)\right] \mid \\
& \leq \frac{\Gamma-\gamma}{2(b-a)}\left[h_{2}\left(a, \frac{3 a+b}{4}\right)+h_{2}\left(x, \frac{3 a+b}{4}\right)+h_{2}\left(x, \frac{a+3 b}{4}\right)+h_{2}\left(b, \frac{a+3 b}{4}\right)\right]
\end{aligned}
$$

for all $\lambda \in[0,1]$ such that $\frac{3 a+b}{4}$ and $\frac{a+3 b}{4}$ are in $T, x \in\left[\frac{3 a+b}{4}, \frac{a+3 b}{4}\right] \cap T$. 


\section{REFERENCES}

[1]. Agarwal R., Bohner M., Peterson A., Inequalities on time scales: a survey, Math. Inequal. Appl., 2001; 4, 535-557.

[2]. Bohner M., Peterson A., Dynamic Equations on Time Scales. An Introduction with Applications, Birkhäuser Boston, Inc., Boston, MA, 2001.

[3]. Bohner M., Peterson A., Advances in dynamic equations on time scales, Birkhäuser Boston, Boston, MA, 2003.

[4]. Bohner M., Matthews T., Ostrowski inequalities on time scales, JIPAM. J. Inequal. Pure Appl. Math., 2008; 9, Article $6,8 \mathrm{pp}$.

[5]. S.S. Dragomir and S. Wang, An inequality of Ostrowski-Grüss type and its applications to the estimation of error bounds for some special means and for some numarical quadrature rules, Comput. Math. Appl., 1997; 33 (11), pp. 16-20.

[6]. Hilger S., Ein Maßkettenkalkül mit Anwendung auf Zentrumsmannigfaltigkeiten, Ph.D. thesis, Universität Würzburg, Würzburg, Germany, 1988.

[7]. Lakshmikantham V., Sivasundaram S., Kaymakcalan B., Dynamic systems on measure chains, Mathematics and its Applications, 370, Kluwer Academic Publishers Group, Dordrecht, 1996.
[8]. Liu W., Tuna A., Jiang Y., On weighted Ostrowski type, Trapezoid type, Grüss type and Ostrowski-Grüss like inequalities on time scales, Applicable Analysis, 2014; Volume 93, Issue 3, 551-571.

[9]. Liu W., Tuna A., Jiang Y., New weighted Ostrowski and Ostrowski-Grüss type inequalities on time scales, Annals of the Alexandru Ioan Cuza University-Mathematics, 2014; Volume LX, Issue 1, 57-76.

[10]. Nwaeze E. R., A new weiğhted Ostrowski type inequality on arbitrary time scale, Journal of King Saud University-Science, 2017; volume 29, number 1, pp. 230-234.

[11]. Ostrowski A., Uber die Absolutabweichung einer differentiierbaren Funktion von ihrem Integralmittelwert, Comment. Math. Helv., 1937; 10, 226-227.

[12]. Tuna A., Daghan D., Generalization of Ostrowski and Ostrowski-Grüss type inequalities on time scales, Comput. Math. Appl., 2010; 60, 803-811.

[13]. Tuna A., Jiang Y., Liu W.J., Weighted Ostrowski, Ostrowski-Grüss and Ostrowski-Čebyšev Type Inequalities on Time Scales, Publ. Math. Debrecen, 2012; 81, 81-10 